\title{
A Comparison Between Two Different Prophylactic Doses of Unfractionated Heparin for Deep Venous Thrombosis Prevention in Laparoscopic Bariatric Surgery
}

\author{
Farzane Ebrahimifard ${ }^{1,2}$, Abdolreza Pazouki ${ }^{1 *}$, Masoud Solaymani Dodaran ${ }^{1}$, Mohammad \\ Vaziri $^{1}$ \\ ${ }^{1}$ Minimally Invasive Surgery Research Center, Rasool Akram Hospital, Tehran University of Medical Sciences, Tehran , IR Iran \\ ${ }^{2}$ Surgical Department, Shahid Beheshti University of Medical Sciences, Tehran, IR Iran
}

\section{A R T I C L E I N F O}

Article type:

Original Article

Article history:

Received: 27 Mar 2012

Revised: 18 Apr 2012

Accepted: 21 Apr 2012

Keywords:

Heparin

Obesity

Bariatric Surgery

Venous Thrombosis

IRCT/NCT registration code:

IRCT201008253384N3

\begin{abstract}
A B S T R A C T
Background: Deep Venous Thrombosis (DVT) is a major risk of morbidity and mortality in morbid obese patients underwent bariatric surgery. There are some controversies in different kind of prophylactic strategies for DVT in laparoscopic bariatric surgeries. Unfractionated heparin (UFH) is an available and reversible anticoagulant used for DVT prophylaxis.

Objectives: This study aimed to compare clinical results of two different dosage regimes of unfractionated heparin for short term prophylaxis of DVT after bariatric surgery. Patients and Methods: 139 patients with morbid obesity who underwent laparoscopic bariatric surgery (laparoscopic Roux-en-Y gastric bypass, sleeve gastrectomy, and laparoscopic gastric banding) were evaluated in two groups: group A received 5000 IU unfractionated heparin q12h and group B received the same dose but q8h ( preliminary dose received before induction of anesthesia followed by 2 or 3 times daily). All patients were evaluated by physical examination and Doppler ultra sound for DVT before and 10 days after surgery.

Results: There was no statistically significant difference between two groups in venous thrombosis. No thrombotic events were observed before and after operations. There were no heparin induced thrombocytopenia and no meaningful difference between two groups in postoperative bleeding.

Conclusions: This study showed that in combination with non-pharmacologic methods for prevention of thromboembolic events, both regimes of UFH prophylaxis had similar clinical effects.
\end{abstract}

Implication for health policy/practice/research/medical education:

This study shows that heparin prescription twice a day is as effective as 3 times daily and there is no need for more doses. Study of this article could be useful for Laparoscopic and Bariatric surgeon.

Please cite this paper as:

Ebrahimifard F, Pazouki A, Solaymani Dodaran M, Vaziri M. A Comparison Between Two Different Prophylactic Doses of Unfractionated Heparin for Deep Venous Thrombosis Prevention in Laparoscopic Bariatric Surgery. J Minim Invasive Surg Sci. $2012 ; 1(2)$ : 58-61. DOI: $10.5812 /$ jmiss.4991

\footnotetext{
* Corresponding author: Abdolreza Pazouki, Minimally Invasive Surgery Research Center, Rasool Akram Hospital, Tehran University of Medical Sciences, Tehran , IR Iran. Tel: +98-2166507055, Fax: +98-21 66501113, E-mail: apazouki@yahoo.com

DOI:10.5812/jmiss.4991

Copyright (c) 2012, Minimally Invasive Surgery Research Center and Mediterranean \& Middle Eastern Endoscopic Surgery Association. This is an open-access article distributed under the terms of the Creative Commons Attribution License, which permits unrestricted use, distribution, and reproduction in any medium, provided the original work is properly cited.
}

\section{Background}

Venous Thromboembolism (VTE) is a major cause of morbidity and mortality in morbid obese patients underwent bariatric surgery (1). Fatal pulmonary emboli (PE) is a leading cause of post-operative death in morbid obese patients. The co-incidence of deep vein thrombosis 
(DVT) and PE following bariatric surgery is $2 \%$ (2). As the number of bariatric operations is rapidly growing, more persons are the at risk of complications of the surgery (3-5). Since diagnosis of venous thrombosis in severely obese patients due to difficulties of performing physical examination is challenging, preventing the patients from venous thromboembolism is crucial in postoperative management of bariatric surgery(6). Several prophylactic measures including unfractionated heparin, lowmolecular weight heparin (LMWH), intermittent compression devices, elastic stockings, early ambulation, and Inferior Vena Cava (IVC) filters have been proposed to reduce high incidence of DVT in postoperative bariatric surgical patients $(7,8)$. Although all clinicians agree that prophylaxis is crucial, still there are some controversies over the best practice for prevention of DVT during laparoscopic surgery $(2,9)$. Based on SAGES (Surgical Association of Gastroenterologist Surgeon) guidelines the prophylactic therapy should be employed to the individual patient who is likely at the risk of VTE but there may be a specific procedure that has a variety of treatment strategies (10).

\section{Objectives}

The study aimed tocompare between clinical results of two different dosage regimes of unfractionated heparin for short term prophylaxis of deep venous thrombosis after bariatric surgery.

\section{Patients and Methods}

This study is a prospective randomized controlled trial(Irct ID: IRCT201008253384N3) performed on 139 patients who underwent laparoscopic bariatric surgery (gastric bypass, sleeve gastrectomy, gastric banding, or gastric plication). All patients were referred to obesity center of Rassool Akram hospital and were selected for laparoscopic bariatric surgery if BMI $>40 \mathrm{~kg} / \mathrm{m}^{2}$, or BMI $>35 \mathrm{~kg} / \mathrm{m}^{2}$ accompanied with comorbidities of diabetes, hypertension, sleep apnea, osteoarthritis, and other obesity induced diseases. Deep and superficial venous system of lower extremities was evaluated by physical examination and color doppler ultrasound before and 10 days after operation. Patients were randomly divided into two groups: group A administered by UFH/5000 IU SC q12h and group B by UFH/5000 IU SC q8h. All patients wore prophylactic compression stockings (below- knee elastic stockings, before and one month after operation) and were ambulated first day post operation. All operations were accomplished through similar technique by several surgeons on the same experience level. Since DVT develops more frequently within first 10 postoperative days we targeted this period for next evaluation. The results examined by Pearson Chi-Square test.

Exclusion criteria included patients with history of DVT and PE (Pulmonary Emboli), evidence of DVI (Deep Venous Insufficiency) in physical exam or Doppler, any bleeding tendency (in history and lab tests), or history of allergy to heparin. Ethical principles were considered in study.

\section{Results}

There were a total number of 139 patients randomly divided into two study groups: 68 patients (48.9\%) in group A received 5000 IU unfractionated heparin subcutaneously q12h, and 71 patients (51.1\%) in group B received $5000 \mathrm{IU}$ in the same route q8h. All operations were performed laparoscopically as a primary bariatric surgery. 126 (90.6\%) patients were female and 13 (9.4\%) patients were male. The mean age, weight, and BMI were 36.74 $\pm 9.5,119.7 \pm 20.3$, and $44.29 \pm 6.9$, respectively without statistically significant difference between two groups (Table 1). More than 72\% (101 patients) underwent laparoscopic Roux-en-Y gastric bypass, while 35 patients (25.2\%) experienced sleeve gastrectomy, 2 patients $(1.4 \%)$ gastric banding, and one patient gastric plication (0.7\%). Twenty one patients (15.1\%) exhibited varicose veins in pre-operative physical examination. Doppler performed before surgery recognized saphenofemoral junction reflux in 2 patients. Mean operative time was $112.5 \pm 35$ without significant difference between two groups (Table 2). There was no correlation between oral contraceptive pill (OCP), hypertension (HRT), or other drugs with post-operative DVT. No relation was found between histories of DVT in patients or their relatives, cardiovascular diseases, blood dyscrasia, or hypertension and post-operative DVT. (No patient had blood dyscrasia).

Group A patients were not statistically different from group B in physical exam and Doppler results before and 10 days after surgery. No DVT occurred on 10th day post operation in both groups (100\% without DVT).

There were three cases of heparin discontinuation due to the bleeding, 2 patients (2.9\%) in group A and one patient $(1.4 \%)$ in group B with no significant difference between groups. Two patients (one in group A and one in group B) underwent reoperation because of hemody-

\begin{tabular}{lllll}
\hline Table 1. Descriptive Statistics & & & & \\
\hline & Patients, No. (\%) & Minimum & Maximum & Mean \pm SD \\
\hline Age & 139 & 17.00 & 68.00 & $36.7482 \pm 9.56980$ \\
Weight & 139 & 89.00 & 200.00 & $119.7158 \pm 20.32829$ \\
Height & 139 & 149.00 & 185.00 & $163.7698 \pm 7.26174$ \\
BMI & 139 & 32.00 & 80.12 & $44.2936 \pm 6.89119$ \\
\hline
\end{tabular}




\begin{tabular}{|c|c|c|c|}
\hline & Patients, No. (\%) & Valid Percent & Cumulative Percent \\
\hline \multicolumn{4}{|l|}{ Gender } \\
\hline Female & $126(90.6)$ & - & - \\
\hline Male & $13(9.4)$ & - & - \\
\hline \multicolumn{4}{|l|}{ Type of Operation } \\
\hline Gastric Bypass & $101(72.7)$ & 72.7 & 72.7 \\
\hline Gastric Sleeve & $35(25.2)$ & 25.2 & 97.8 \\
\hline Gastric Banding & $2(1.4)$ & 1.4 & 99.3 \\
\hline Plication & $1(0.7)$ & 0.7 & 100.0 \\
\hline Total & $139(100.0)$ & 100.0 & \\
\hline \multicolumn{4}{|c|}{ Physical Exam of Legs for DVT Before Surgery } \\
\hline Normal & $135(97.1)$ & 97.1 & 97.1 \\
\hline Evidence of DVT & $1(0.7)$ & 0.7 & 97.8 \\
\hline DVI & $3(2.2)$ & 2.2 & 100.0 \\
\hline Total & $139(100.0)$ & 100.0 & \\
\hline \multicolumn{4}{|c|}{ Physical Exam of Legs for DVT After Surgery } \\
\hline Normal & $137(98.6)$ & 98.6 & 98.6 \\
\hline Evidence of DVT & $1(0.7)$ & 0.7 & 99.3 \\
\hline DVI & $1(0.7)$ & 0.7 & 100.0 \\
\hline Total & $139(100.0)$ & 100.0 & \\
\hline \multicolumn{4}{|c|}{ Doppler Before Surgery } \\
\hline Normal & $138(99.3)$ & 99.3 & 99.3 \\
\hline DVI & $1(0.7)$ & 0.7 & 100.0 \\
\hline Total & $139(100.0)$ & 100.0 & \\
\hline \multicolumn{4}{|c|}{ Doppler After Surgery } \\
\hline Normal & $139(100.0)$ & 100.0 & 100.0 \\
\hline
\end{tabular}

namic instability and anemia and the another one was managed conservatively. The etiology of bleeding in reoperated patients was stapler line arterial hemorrhage which was controlled laparoscopically.

\section{Discussion}

Morbid obesity is a significant and rapidly growing problem in modern world. Severe obesity is attributed to numerous adverse effects that cause earlier death (11). For severely obese patients (BMI $>40 \mathrm{~kg} / \mathrm{m}^{2}$, or BMI $>35$ $\mathrm{kg} / \mathrm{m}^{2}$ with associated comorbidity) who fail to achieve weight loss through other conservative treatments, bariatric surgery is a recommended treatment. Morbid obesity has been shown as a major independent risk factor for acute pulmonary emboli (12). Also bariatric surgery, in particular laparoscopic surgery, is a major intervention related to development of Postoperative venous thromboembolism and fatal PE $(2,9)$. Although Erickson et al. recommended thromboprophylaxis to all operated obese patients, Caprini and colleagues described multimodality risk reduction protocol based on each patient before surgery $(5,13)$. Wille-Jorgensen and Ott identified that the combination of compression stockings and low molecular weight heparin was more effective than either alone (14). F. Kalfarentzos and colleagues in a prospective study evaluated the effects of 2 different doses of nadroparin as DVT prophylaxis in 60 open bariatric surgery and the results indicated that the dose of $5700 \mathrm{IU}$ (i.e. lower dose) once daily was effective, safe, well-tolerated, and therefore recommended for venous thromboembolism (2). In another survey, Scholten et al. studied 481 patients divided into 2 groups and underwent bariatric surgery and concluded that the use of higher dose of enoxaparin (40 mg q12h) was more effective in lowering DVT complications without any bleeding (9). We examined effects of two doses of unfractionated heparin for DVT prophylaxis in morbid obese patients underwent laparoscopic bariatric surgery. Unfractionated heparin is an available, reversible, and cost effective anticoagulant which is recommended for DVT prophylaxis. There was no need to check lab test for its efficacy (as X factor for LMWH).

This study shows that unfractionated heparin twice daily was as sufficient, safe, well tolerated, and convenient as three times per day for DVT prophylaxis in laparoscopic bariatric surgery. Although other studies showed total incidence of thromboembolism including subclinical cases is obviously much higher than clinical cases (5), in this study there was no statistically difference between 
results of physical examination and Doppler evaluation before and after surgery. Since early ambulation and compression stockings were employed in all patients, a multimodality strategy on prophylaxis of venous thrombosis should be considered. However, a comparison between non-pharmacological methods and heparin derivatives medication in DVT prophylaxis is suggested. This study shows that both regimes of UFH prophylaxis in combination with non-pharmacologic methods for prevention of thromboembolic events, have similar clinical effects.

\section{Acknowledgments}

We would like to appreciate Dr. S. Mokhber, Dr. Z. Tamannaie, and Ms. Pishgah for their kind cooperation.

\section{Authors' Contribution}

First author and corresponding author contributed $100 \%$ and the other authors $50 \%$.

\section{Financial disclosure}

The authors declare no financial disclosure.

\section{Funding/Support}

This research has been supported by Tehran University of Medical Sciences and Health Services.

\section{References}

1. Gonzalez QH, Tishler DS, Plata-Munoz JJ, Bondora A, Vickers SM, Leath $\mathrm{T}$, et al. Incidence of clinically evident deep venous thrombosis after laparoscopic Roux-en-Y gastric bypass. Surg Endosc.
2004;18(7):1082-4.

2. Kalfarentzos F, Stavropoulou F, Yarmenitis S, Kehagias I, Karamesini M, Dimitrakopoulos A, et al. Prophylaxis of venous thromboembolism using two different doses of low-molecularweight heparin (nadroparin) in bariatric surgery: a prospective randomized trial. Obes Surg. 2001;11(6):670-6.

3. Blaszyk H, Wollan PC, Witkiewicz AK, Bjornsson J. Death from pulmonary thromboembolism in severe obesity: lack of association with established genetic and clinical risk factors. Virchows Arch.1999;434(6):529-32.

4. Brasileiro AL, Miranda F, Jr., Ettinger JE, Castro AA, Pitta GB, de Moura LK, et al. Incidence of lower limbs deep vein thrombosis after open and laparoscopic gastric bypass: a prospective study. Obes Surg. 2008;18(1):52-7.

5. Eriksson S, Backman L, Ljungstrom KG. The incidence of clinical postoperative thrombosis after gastric surgery for obesity during 16 years. Obes Surg. 1997;7(4):332-5; discussion 6.

6. Markel A, Weich Y, Gaitini D. Doppler ultrasound in the diagnosis of venous thrombosis. Angiology. 1995;46(1):65-73.

7. Cotter SA, Cantrell W, Fisher B, Shopnick R. Efficacy of venous thromboembolism prophylaxis in morbidly obese patients undergoing gastric bypass surgery. Obes Surg. 2005;15(9):1316-20.

8. Michota FA. Prevention of venous thromboembolism after surgery. Cleve Clin J Med. 2009;76(Suppl 4):S45-52.

9. Scholten DJ, Hoedema RM, Scholten SE. A comparison of two different prophylactic dose regimens of low molecular weight heparin in bariatric surgery. Obes Surg. 2002;12(1):19-24.

10. Society of American Gastrointestinal and Endoscopic Surgeons. Guidelines for Deep Venous Thrombosis Prophylaxis During Laparoscopic Surgery. Los Angeles: SAGES; 2006; Available from: http://www.sages.org/publication/id/C/.

11. Monteforte MJ, Turkelson CM. Bariatric surgery for morbid obesity. Obes Surg. 2000;10(5):391-401.

12. Blaszyk H, Bjornsson J. Factor V leiden and morbid obesity in fatal postoperative pulmonary embolism. Arch Surg. 2000;135(12):1410-3.

13. Caprini JA, Arcelus JI, Hasty JH, Tamhane AC, Fabrega F. Clinical assessment of venous thromboembolic risk in surgical patients. Semin Thromb Hemost. 1991;17(Suppl 3):304-12.

14. Wille-Jorgensen P, Ott P. Predicting failure of low-dose prophylactic heparin in general surgical procedures. Surg Gynecol Obstet. 1990;171(2):126-30. 